\title{
Interactive comment on "Evapotranspiration
} partition using the multiple energy balance version of the ISBA-A-g $\mathrm{g}_{\mathrm{s}}$ land surface model over two irrigated crops in a semi-arid Mediterranean region (Marrakech, Morocco)" by Ghizlane Aouade et al.

Ghizlane Aouade et al.

ghizlane.aouade@gmail.com

Received and published: 26 March 2020

We thank the reviewer for his in depth reading of the manuscript. All the typo and grammar errors reported in the annotated manuscript have been corrected. In addition, you will find below the answer to his comments.

1.1. "while water transfers are active on the $0-2 m$ layer only" reformulate 
OK. As the discretization of the soil column up to $12 \mathrm{~m}$ in ISBA is a trick to better predict the soil temperature profile, the second part of the sentence has simply been removed.

1.2. "Indeed, the appearance of a strong sensitivity of the two parameters and, seems to be consistent (Choudhury and Idso 1985) and optimal higher (lower) values than literature of ( ) are obtained (Table 3)." reformulate

OK. Done as follows:

"Indeed, the strong sensitivity of the two parameters and, seems to be consistent with Choudhury and Idso (1985). In addition, the optimal value of ( ) is higher (lower) than literature (table 3)."

1.3. "As a conclusion, the high sensitivity to the new parameters introduced in ISBAMEB and the optimal values of the sensitive parameters being significantly different from default literature values, means that sstudies at the local scales should be duplicated to determine specific parameters values for for different eco- and agro-systems to feed the ECOCLIMAP-II data base with specific parameter values in the view of a large-scale applications." Unclear conclusion.

OK. Reworded as follows:

"As a conclusion, the optimal values of the sensitive parameters being significantly different from literature values, studies at the local scales should be duplicated to determine specific parameters values for for different eco- and agro-systems in the view of a large-scale applications."

1.4. "This is probably because the bare soil area between the tree rows (the inter-row is about $8 \mathrm{~m}$.) is not sufficiently large to consider two independent heat sources located side by side." Clarify this sentence. OK. Reworded as follows:

Printer-friendly version

"This is probably because the bare soil area between the tree rows (the inter-row is about $8 \mathrm{~m}$.) is not sufficiently large to consider that soil and vegetation heat sources doesn't interact with each other by locating the two sources side by side."

Discussion paper 
1.5. "It is well known that part of the observed discrepancies between simulations and observations can be related to the eddy eddy-covariance measurements because of the associated strong heterogeneity within the footprint during an irrigation event." well the models are not super trustworthy neither - nuance that more.

Agree. Of course. The sentence has been moved at the end of the paragraph to complement the analysis of the model deficiencies around an irrigation events and it has been reworded as follows:

"In addition to the model deficiencies at the time of irrigation as already highlighted, part of the discrepancies between simulations and observations can be related to the eddy eddy-covariance measurements because of the associated strong heterogeneity within the footprint during an irrigation event."

1.6. "... dissipates much less energy by soil conduction compared to the other two configurations" Why?

OK. The explanation was given in the next paragraph. This is now explained as follows:

"... dissipates much less energy by soil conduction compared to the other two configurations. This is due to a compensation between the soil and the vegetation patches as explained below."

1.7. "As for the latent heat flux, the dual sources configurations outperformed the single source version for sensible heat flux predictions over complex cover with wide differences in performances between ISBA-1P on one hand, and ISBA-2P and ISBAMEB on the other hand over the olive orchard site (Figure 5)." Unclear sentence.

OK. Sentence reworded as follows:

"For the sensible heat fluxes, the dual sources configurations ISBA-2P and ISBA-MEB also outperformed the single source version ISBA-1P for sensible heat flux predictions over the olive orchard." 
1.8. "... it appears that the patch bare soil dissipates a lot of energy by conduction as shown by the ..." A lot compared to what?

OK. Sentence reworded as follows:

"... it appears that the patch bare soil (like ISBA-1P) dissipates much more energy by conduction than ISBA-MEB as shown by the ..."

1.9. "The most striking feature is the significantly higher energy available for convection and conduction at the soil level for ISBA-1P and ISBA-2P with regards to ISBA-MEB (the reverse is obviously true for vegetation net radiation, not shown)." clarify waht is meant by conduction and convdction - unclear

Agree. Sentence reworded to make it clearer as follows:

"The net radiation at the soil surface is obviously lower for ISBA-MEB than for ISBA-1P and $-2 \mathrm{P}$ because of vegetation screening and real partition between the two sources."

1.10. "ISBA-1P lies in-between because the canopy is open ( $\mathrm{FC}=55 \%)$." reformulate sentence

The sentence was discarded in the new version of the manuscript without changing the conclusion of the analysis.

1.11. "The transpiration measured by the Sapflow at the Olive orchard site was aggregated at a daily timescale and converted in $\mathrm{mm} /$ day." only sap velocity is measured explain how you convert to transpiration

Agree. According to the reviewer's comment (and also in response to reviewer 1), the extrapolation from sapflow measurements to transpiration at the field scale was detailed at 2.1.4 Data description section "evapotranspiration partition".

Interactive comment on Hydrol. Earth Syst. Sci. Discuss., https://doi.org/10.5194/hess-2019532, 2019. 